\title{
NOUVELLE
}

\section{La télomérase:}

un antigène tumoral universel pour l'immunothérapie

\section{anti-cancéreuse}

Olivier Adotévi, Francisco Garcia Pons,

Pierre Langlade-Demoyen
0. Adotévi: Unité immunité

cellulaire anti-virale,

Institut Pasteur,

25, rue du Dr Roux,

75015 Paris, France et Inserm

U.255, Immunologie

cellulaire et clinique,

Institut Biomédical

des cordeliers,

15, rue de l'દ́cole

de Médecine,

> Les lymphocytes $\mathrm{T} \mathrm{CD8}{ }^{+}$cytotoxiques (LTC) représentent l'un des effecteurs majeurs de l'immunité tumorale [1]. Ainsi de nombreuses stratégies d'immunothérapie anti-tumorale ont pour objectif d'induire ou d'augmenter l'activité des LTC spécifiques de tumeur chez les patients atteints de cancers. Les LTC sont des cellules spécialisées du système immunitaire qui reconnaissent, grâce à des récepteurs spécifiques d'antigènes, des petits peptides de neuf à dix acides aminés présentés par des molécules du complexe majeur d'histocompatibilité de classe I (CMH I) à la surface des cellules tumorales. La reconnaissance par les LTC des complexes CMH I-peptides peut conduire à la lyse de la cellule tumorale d'où leur nom de «cellules tueuses». La plupart des peptides reconnus par les LTC dérivent de protéines présentes dans les cellules tumorales, appelées «antigènes associés aux tumeurs » ou tumor-associated antigens (TAA). Au cours de cette dernière décennie, l'identification de ces antigènes associés aux tumeurs a représenté un tournant décisif dans l'immunothérapie des cancers et a permis d'envisager des protocoles de vaccination à l'aide de ces antigènes définis moléculairement [2].

Cependant, la plupart des antigènes identifiés à ce jour sont des protéines dont l'expression est restreinte à certains types de cancers, ce qui réduit les applications potentielles d'immunothérapie à un nombre limité de cancers. Des efforts ont été réalisés pour découvrir de

nouveaux antigènes tumoraux, plus largement exprimés, ce qui permettrait de cibler plusieurs types de cancers. C'est ainsi que récemment, on a proposé une enzyme, appelée télomérase, comme premier membre d'une nouvelle classe d'antigènes tumoraux exprimés par de nombreux cancers.

La télomérase est une ribonucléoprotéine qui induit la synthèse de I'ADN télomérique, permettant ainsi de maintenir la longueur des télomères et la stabilité des chromosomes $[3,4](\rightarrow)$. Cette enzyme est exprimée précocement durant l'ontogénie et dans certaines cellules qui ont un

$(\rightarrow) \mathrm{m} / \mathrm{s}$ $2000, n^{\circ} 4$, p. 473 et p. 547 pouvoir de réplication élevé comme les cellules souches hématopoïétiques [5, 6]. L'activation de la télomérase est suffisante pour induire l'immortalisation cellulaire, événement clé dans le processus de transformation maligne [7]. La sous-unité catalytique de la télomérase appelée transcriptase inverse de la télomérase (hTERT, human telomerase reverse transcriptase) est surexprimée dans plus de $90 \%$ des cancers humains [8, 9]. En comparaison, les cellules normales expriment peu ou pas de hTERT [6]. Cette expression préférentielle de hTERT par les cellules tumorales, a fait de cette protéine une cible très attractive pour les thérapeutiques anticancéreuses [10] $(\rightarrow)$.
Plusieurs équipes dont la nôtre ont démontré récemment que la télomérase pouvait être une source de peptides présents à la surface des cellules tumorales et reconnus par les LTC $[11,12]$. Ainsi, il a été possible d'induire in vitro des LTC en exposant des lymphocytes de donneur sain ou de patients atteints de cancer à des peptides hTERT [12-14]. De plus les LTC anti-hTERT étaient capables de tuer in vitro des cellules tumorales provenant de tissus variés (poumon, sein, peau, prostate, etc.) (Tableau 1). Ces résultats démontrent qu'il existe, dans le répertoire lymphocytaire de sujets sains et de malades atteints d'un cancer, des précurseurs de LTC anti-hTERT. Dans une étude pré-clinique réalisée par notre groupe, des souris transgéniques exprimant les molécules humaines HLA-A2.1 furent immunisées avec les peptides dérivés de hTERT. Ces souris « humanisées» furent capables de produire des réponses cytotoxiques spécifiques dirigées contre des cellules tumorales [12]. D’autre part, il a été démontré que la vaccination de souris à l'aide de cellules dendritiques transfectées avec l'ARN de la télomérase murine, pouvait induire une réponse LTC anti-tumorale capable de protéger les souris vaccinées [15]. L'ensemble de ces études préliminaires in vitro et in vivo sur l'immunogénicité des peptides dérivés de la télomérase ont ouvert des perspectives d'immunothérapie très prometteuses, le système immunitaire étant capable d'apprendre à reconnaître $\rightarrow$ m/s la télomérase et en consé2001, $\mathrm{n}^{\circ} \mathbf{1 0}$ quence d'inhiber la croisp. 1076 sance tumorale. 
Cependant, l'inconvénient majeur de cette approche d'immunothérapie antitélomérase serait la survenue de manifestations auto-immunes chez les patients ainsi traités $[16,17]$. En effet, dans les conditions normales, les cellules du système immunitaire, en particulier les lymphocytes, ont appris à faire la différence entre une protéine du soi (auto-antigène) et un antigène étranger (bactérien, viral, etc.), seul ce dernier devant être éliminé. Ce processus d'éducation des lymphocytes est connu sous le nom de tolérance immunitaire. Dans certaines circonstances, la rupture de cette tolérance peut conduire le système immunitaire à détruire des antigènes $d u$ soi, provoquant une atteinte autoimmune. La télomérase étant un autoantigène, comme le sont la plupart des antigènes tumoraux, les LTC spécifiques induits contre cette enzyme, et destinés à éliminer des cellules tumorales, pourraient aussi s'attaquer à des cellules normales autologues exprimant cette protéine.

Si l'on se réfère aux données actuelles concernant l'immunothérapie anti-télomérase, ce risque d'atteinte auto-immune semble minime. Les études in vitro chez l'homme ont montré que les LTC antitélomérase capables de tuer des cellules tumorales n'ont aucun effet sur les cellules souches hématopoïétiques $\mathrm{CD}_{3} 4^{+}$susceptibles d'exprimer la télomérase [12, 13]. Une expression relativement faible de la télomérase dans ces cellules souches par rapport à celle que l'on observe dans les cellules tumorales ou son expression inconstante pourraient expliquer cette observation [18].

Des arguments plus convaincants nous sont apportés par l'analyse des résultats d'un essai de phase I à l'Institut du Cancer Dana-Farber (États-Unis), dont l'objectif était d'établir la sécurité d'une immunothérapie utilisant des peptides hTERT. Au cours de cette étude qui incluait sept patients atteints de cancers avancés et vaccinés par hTERT, aucun effet secondaire délétère n'a été observé [18].

Enfin notre groupe a récemment identifié de nouveaux peptides dérivés de hTERT, ayant une affinité faible pour les molécules HLA-A2.1. L'utilisation de peptides de faible affinité permet de limiter l'expression de ces peptides aux seules cellules exprimant fortement la télomérase, ce qui sélectionne donc les cellules tumorales. La vaccination des souris transgéniques HLA-A2.1 avec ces peptides de faible affinité n'a entraîné aucune manifestation auto-immune chez ces animaux [19]. Ces résultats confirment des résultats obtenus précédemment dans un autre modèle de souris immunisées avec l'antigène télomérase [15]. Actuellement, des essais de vaccination de phase I chez des patients atteints de cancer de la prostate ont lieu à San Diego (États-Unis), dont les résultats devraient être connus fin $2004 . \diamond$

Telomerase: a tumor antigen

for anticancer immunotherapy

\section{RéFÉRENCES}

1. Pardoll D. T cells and tumors. Nature $2001 ; 411$ : 1010-2.

2. Rosenberg SA. Progress in human tumour immunology and immunotherapy. Nature 2001; 411: 380-4.

3. Blackburn EH. Telomerases. Annu Rev Biochem 1992; 61: 113-29.

4. Counter CM, Avilion AA, LeFeuvre $C \varepsilon$, et al. Telomere shortening associated with chromosome instability is arrested in immortal celles which express telomerase activity. EMBO J 1992; 11: 1921-9.

\begin{tabular}{|c|c|c|c|c|c|}
\hline \multirow{2}{*}{$\begin{array}{l}\text { CELLULES } \\
\text { CIBLES }\end{array}$} & \multirow{2}{*}{$\begin{array}{l}\text { ORIGINE } \\
\text { TUMORALE }\end{array}$} & \multirow{2}{*}{$\begin{array}{c}\text { EXPRESSION }{ }^{a} \\
\text { hTERT }\end{array}$} & \multirow[b]{2}{*}{ HLA-A2 ${ }^{b}$} & \multicolumn{2}{|c|}{ POURCENTAGE DE LySE } \\
\hline & & & & LTCp540d & LTCp865 \\
\hline T2+ peptide & & ND & + & 59 & 48 \\
\hline T2 & & ND & + & 11 & 4 \\
\hline MCF7 & Sein & + & + & 39 & 41 \\
\hline SKBR3 & & + & - & 7 & 9 \\
\hline SW480 & Côlon & + & + & 12 & 37 \\
\hline НСТ011 & & + & - & 9 & 6 \\
\hline $\mathrm{H} 69$ & Poumon & + & + & 41 & 9 \\
\hline H146 & & + & - & 11 & 5 \\
\hline 624 & Mélanome & + & + & 48 & 39 \\
\hline 1351 & & + & - & 12 & 6 \\
\hline LnCap & Prostate & + & + & 44 & 41 \\
\hline Pc3 & & + & - & 9 & 5 \\
\hline
\end{tabular}

Tableau I. Lyse de différentes cellules tumorales par des LTC anti-télomérase dérivés d'un patient atteint de cancer. ${ }^{a}$ Expression de hTERT par les cellules tumorales, déterminée par RT-PCR. ${ }^{b}$ Expression de HLA-A2 mesurée par cytométrie en flux après marquage avec un anticorps monoclonal. ${ }^{\mathrm{C}}$ Cytotoxicité cellulaire mesurée par le test de relargage au ${ }^{51} \mathrm{Cr}$, avec un rapport effecteur/cible de $50: 1 .{ }^{d}$ Les lignées LTC p540 et LTC p865 de patient obtenues par immunisation avec les peptides HTERT p540 et p865 respectivement. ND: non déterminé (d'après [12]). 
5. Kim NW, Piatyszek MA, Prowse KR, et al. Specific association of human telomerase activity with immortal celles an cancer. Science 1994; 266: 2011-5.

6. Broccoli D, Young JW, de Lange T. Telomerase activity in normal an malignant hematopoietic cells. Proc Natl Acad Sci USA 1995; 92: 9082-6.

7. Meyerson M, Counter CM, Eaton $\varepsilon N$, et al. hEST2, the putative human telomerase catalytic subunit gene, is up-regulated in tumor celles an during immortalization. Cell 1997; 90: 785-95.

8. Kim NW. Clinical implications of telomerase in cancer. Eur J Cancer 1997; 33: 781-6.

9. Nakamura TM, Morin GB, Chapman KB, et al. Telomerase catalytic subunit homologs from fission yeast an human. Science 1997; 277: 955-9.
10. Shay JW, Wright WE. Telomerase: a target for cancer therapeutics. Cancer Cell 2002; 2: 257-65.

11. Vonderheide RH, Hahn WC, Schultze JL, Nadler LM. The telomerase catalytic subunit is a widely expressed tumorassociated antigen recognized by cytotoxic $T$ lymphocytes. Immunity 1999; 10: 673-9.

12. Minev B, Hipp J, Firat $H$, et al. Cytotoxic T cell immunity against telomerase reverse transcriptase in humans. Proc Natl Acad Sci USA 2000; 97 : 4796-801.

13. Vonderheide RH, Anderson KS, Hahn WC, Butler MO, Schultze JL, Nadler LM. Characterization of HLAA3-restricted cytotoxic $T$ lymphocytes reactive against the widely expressed tumor antigen telomerase. Clin Cancer Res 2001; 7 : 3343-8.
14. Vonderheide RH, Schultze $J$, Anderson KS, et al. Equivalent induction of telomerase-specific cytotoxic T lymphocytes from tumor-bearing patients and healthy individuals. Cancer Res 2001; 61: 8366-70.

15. Nair SK, Heiser A Boczkowski D, et al. Induction of cytotoxic $T$ cell responses an tumor immunity against unrelated tumors using telomerase reverse transcriptase RNA transfected dendritic cells. Nat Med 2000; 6: 1011-7.

16. Nanda NK,Sercarz $\varepsilon \varepsilon$. Induction of anti-selfimmunity to cure cancer. Cell 1995 14; 82: 13-7.

17. Houghton AN. Cancer antigens: immune recognition of self an altered self. J Exp Med 1994 ; 180: 1-4.
18. Vonderheide RH. Telomerase as a universel tumor-associated antigen for cancer immunotherapy. Oncogene 2002; 21 : 674-9.

19. Hernandez J, Garcia-Pons F, Lone $\mathrm{YC}$, et al.

Identification of a human telomerase reverse transcriptase peptide of low affinity for HLA A2.1 that induces cytotoxic $T$ lymphocytes and mediates lysis of tumor celles. Proc Natl Acad Sci USA 2002; 99: 12275-80.

\section{NOUVELLE}

\section{Contrôler l'équilibre redox cellulaire: une fonction ubiquitaire pour la protéine prion cellulaire?}

Benoît Schneider, Mathéa Pietri, Myriam Ermonval, Sophie Mouillet-Richard, Odile Kellermann

> Les encéphalopathies spongiformes subaiguës transmissibles (ESST) constituent un groupe de maladies neurodégénératives qui provoquent inéluctablement la mort après une période d'incubation longue [1]. L'agent pathogène responsable des ESST - tremblante du mouton ou scrapie, ESB ou maladie de la vache folle et maladie de Creutzfeldt-
Jakob chez l'homme - est composé essentiellement, voire exclusivement, d'une protéine nommée PrPSc pour forme scrapie ( $\mathrm{Sc}$ ) de la protéine prion cellulaire ou $\operatorname{PrPC}$. Ces deux isoformes possèdent la même structure primaire mais des conformations différentes. La conversion de la PrPC en sa forme scrapie serait à l'origine de la maladie.
Laboratoire Différenciation

cellulaire et Prions, Cnrs

UPR 1983 et Institut Pasteur,

7, rue Guy Môquet,

94801 Villejuif, France.

bschneid@vjf.cnrs.fr 